\title{
THE CURVATURES OF SOME SKEW FUNDAMENTAL FORMS
}

\author{
TILLA KLOTZ MILNOR
}

\begin{abstract}
Fix a unit normal vector field on a surface $C^{4}$-immersed in a Riemannian 3-manifold of constant sectional curvature. Suppose $H$ and $K$ are mean and Gauss curvatures respectively, and that $H^{\prime}=\sqrt{H^{2}-K}$. Wherever $H^{\prime} \neq 0$, define $\mathrm{I}^{\prime}$, II' and III' by $H^{\prime} \mathrm{I}^{\prime}=\mathrm{II}-H \mathrm{I}, H^{\prime} \mathrm{II}^{\prime}=H \mathrm{II}-$ $K \mathrm{I}$ and $\mathrm{III}^{\prime}=H \mathrm{II}^{\prime}-K \mathrm{I}^{\prime}$, where $\mathrm{I}$ and $\mathrm{II}$ are the first and second fundamental forms. For constants $\alpha, \beta$, and $\gamma$, let $\Lambda^{\prime}=\alpha I^{\prime}+\beta I^{\prime}+\gamma I^{\prime} I^{\prime}$. Wherever $H^{\prime} \neq 0$ and $\Lambda^{\prime}$ is nondegenerate, the curvature of this (not necessarily Riemannian) metric $\Lambda^{\prime}$ is computed in terms of $K\left(\mathrm{I}^{\prime}\right)$ and more familiar quantities on the surface. Some discussion of $K\left(\mathrm{I}^{\prime}\right)$ is also included.
\end{abstract}

1. Suppose a unit normal vector field is fixed on a surface $S$ which is $C^{2}$-immersed in a Riemannian 3-manifold $\Re$. Then the mean and extrinsic curvatures $H$ and $K$ are well defined on $S$, as are the fundamental forms I, II, and III $=2 H$ II $-K$ I. (See [2].) Whenever $H^{\prime}=\sqrt{H^{2}-K} \neq 0$, one can also consider the skew fundamental forms $\mathrm{I}^{\prime}, \mathrm{II}^{\prime}$ and III' given by $H^{\prime} \mathrm{I}=\mathrm{II}-$ $H \mathrm{I}, H^{\prime} \mathrm{II}^{\prime}=H \mathrm{II}-K \mathrm{I}$ and $\mathrm{III}^{\prime}=H \mathrm{II}^{\prime}-K \mathrm{I}^{\prime}$. For a fuller discussion of skew forms, see [10].

In this paper, we assume that $\mathfrak{T}$ has constant sectional curvature, and obtain an expression for the curvature $K\left(\Lambda^{\prime}\right)$ of any linear combination $\Lambda^{\prime}=\alpha I^{\prime}+\beta I^{\prime}+\gamma I^{\prime} I^{\prime}$ with constant coefficients. This gives $K\left(\Lambda^{\prime}\right)$ in terms of $K\left(\mathrm{I}^{\prime}\right)$, and more ordinary quantities on $S$. A brief discussion of $K\left(\mathrm{I}^{\prime}\right)$ appears in $\$ 4$.

Information about skew fundamental forms is potentially useful in studying surfaces in-the-large. For these forms are globally available on any surface which is umbilic-free (although one must work on the universal covering surface in nonorientable cases). An example of an open conjecture involving such surfaces can be found in [7] or [8].

The methods and results here are strikingly similar to those in [9], where the curvature $K(\Lambda)$ of any linear combination $\Lambda=\alpha I+\beta I I+\gamma I I I$ with constant coefficients is computed, assuming again that $\mathfrak{T}$ has constant sectional curvature. This similarity is not surprising, since in terms of linesof-curvature coordinates $x, y$ on $S$,

Received by the editors July 1, 1975.

AMS (MOS) subject classifications (1970). Primary 53B25, 53B30; Secondary 53A05, 53A35. 


$$
\begin{aligned}
\mathrm{I} & =E d x^{2}+G d y^{2}, & \mathrm{I}^{\prime} & =-E d x^{2}+G d y^{2}, \\
\mathrm{II} & =k_{1} E d x^{2}+k_{2} G d y^{2}, & \mathrm{II}^{\prime} & =-k_{1} E d x^{2}+k_{2} G d y^{2}, \\
\mathrm{III} & =k_{1}^{2} E d x^{2}+k_{2}^{2} G d y^{2}, & \mathrm{III}^{\prime} & =-k_{1}^{2} E d x^{2}+k_{2}^{2} G d y^{2},
\end{aligned}
$$

where the principal curvatures $k_{1}$ and $k_{2}$ are indexed with $k_{1} \leqslant k_{2}$ so that $K=k_{1} k_{2}, 2 H=k_{1}+k_{2}$ and $2 H^{\prime}=k_{2}-k_{1}$.

2. For simplicity, assume that $S$ is an umbilic-free surface $C^{\infty}$ immersed in a $C^{\infty}$ Riemannian 3-manifold $\Re$. Then $H^{\prime}$ never vanishes, and $C^{\infty}$ lines-ofcurvature coordinates $x, y$ are locally available anywhere on $S$. In terms of such coordinates, (1) holds, and $K\left(\mathrm{I}^{\prime}\right)$ is given by

$$
4(E G)^{2} K\left(\mathrm{I}^{\prime}\right)=-G_{x}(E G)_{x}+E_{y}(E G)_{y}+2 E G\left(G_{x x}-E_{y y}\right) .
$$

If $\alpha, \beta$ and $\gamma$ are constants the (not necessarily Riemannian) metric $\Lambda^{\prime}=\alpha \mathrm{I}^{\prime}$ $+\beta \mathrm{II}^{\prime}+\gamma \mathrm{III}^{\prime}$ is nondegenerate so long as $\varepsilon_{1} \varepsilon_{2} \neq 0$, where $\varepsilon_{1}=\alpha+\beta k_{1}+$ $\gamma k_{1}^{2}$ and $\varepsilon_{2}=\alpha+\beta k_{2}+\gamma k_{2}^{2}$. The curvature $K\left(\Lambda^{\prime}\right)$ of $\Lambda^{\prime}$ is given wherever $\varepsilon_{1} \varepsilon_{2} \neq 0$ by

$$
\begin{aligned}
4\left(\varepsilon_{1} \varepsilon_{2} E G\right)^{2} K\left(\Lambda^{\prime}\right)= & -\left(\varepsilon_{2} G\right)_{x}\left(\varepsilon_{1} \varepsilon_{2} E G\right)_{x}+\left(\varepsilon_{1} E\right)_{y}\left(\varepsilon_{1} \varepsilon_{2} E G\right)_{y} \\
& +2 \varepsilon_{1} \varepsilon_{2} E G\left[\left(\varepsilon_{2} G\right)_{x x}-\left(\varepsilon_{1} E\right)_{y y}\right] .
\end{aligned}
$$

Because $\Re$ has constant sectional curvature, the Codazzi-Mainardi equations are the same as those $[12$, p. 111] which apply when $\mathfrak{N}$ is Euclidean 3 -space $E^{3}$. Using the given coordinates $x, y$, the Codazzi-Mainardi equations reduce to

$$
\left(k_{2}\right)_{x} G=-H^{\prime} G_{x}, \quad\left(k_{1}\right)_{y} E=H^{\prime} E_{y} .
$$

Thus, if $\varepsilon=\alpha+\beta H+\gamma K$, we have

$$
\left(\varepsilon_{2} G\right)_{x}=\varepsilon G_{x}, \quad\left(\varepsilon_{1} E\right)_{y}=\varepsilon E_{y} .
$$

Substituting (5) in (3), and using the values for $G_{x}$ and $E_{y}$ provided by (4), we get

$4\left(\varepsilon_{1} \varepsilon_{2}\right)^{2} K\left(\Lambda^{\prime}\right)=4 \varepsilon \varepsilon_{1} \varepsilon_{2} K\left(\mathrm{I}^{\prime}\right)$

$$
\begin{aligned}
+\left(\beta^{2}-4 \alpha \gamma\right)\{ & \frac{d k_{2}}{d s_{1}}\left[(\beta+\gamma H) K \frac{d}{d s_{1}} \ln \left|\frac{k_{1}}{k_{2}}\right|+\gamma H^{\prime} \frac{d K}{d s_{1}}-2 \alpha \frac{d H^{\prime}}{d s_{1}}\right] \\
& \left.+\frac{d k_{1}}{d s_{2}}\left[(\beta+\gamma H) K \frac{d}{d s_{2}} \ln \left|\frac{k_{1}}{k_{2}}\right|+\gamma H^{\prime} \frac{d K}{d s_{2}}-2 \alpha \frac{d H^{\prime}}{d s_{2}}\right]\right\},
\end{aligned}
$$

where $d / d s_{1}=(1 / \sqrt{E}) \partial / \partial x$ and $d / d s_{2}=(1 / \sqrt{G}) \partial / \partial y$ are unit tangent vector fields in the principal directions $d y \equiv 0, d x>0$ and $d x \equiv 0, d y>0$ respectively. Had we assumed $k_{1}>k_{2}$, a minus sign would appear before the factors $(\beta+\gamma H)$ in (6).

If $S$ is only $C^{4}$-immersed in $\mathfrak{N}$, the derivation of (6) is not valid, since lines-of-curvature coordinates need only be $C^{2}$ smooth. But near any point $p$ 
at which $\varepsilon_{1} \varepsilon_{2} \neq 0, K\left(\Lambda^{\prime}\right)$ is defined, and we can approximate $S$ by a $C^{\infty}$-immersed surface $\hat{S}$ so that partial derivatives up through order four of the two immersions coincide at $p$. Then $K\left(\Lambda^{\prime}\right)$ is the same for $S$ as for $\hat{S}$ at $p$, and (6) applies to compute $K\left(\Lambda^{\prime}\right)$ on $\hat{S}$. But all quantities in (6) computed for $\hat{S}$ at $p$ equal their counterparts on $S$ at $p$. Thus (6) holds at $p$ on $S$ as well. This gives our main result.

TheOREM. Let $S$ be a surface $C^{4}$-immersed in a $C^{\infty}$ 3-manifold of constant sectional curvature. Let $\alpha, \beta$ and $\gamma$ be constants. At any nonumbilic where $\varepsilon_{1} \varepsilon_{2} \neq 0$, the curvature of the metric $\Lambda^{\prime}=\alpha \mathrm{I}^{\prime}+\beta \mathrm{II}^{\prime}+\gamma \mathrm{III}^{\prime}$ is given by (6), with the principal curvatures indexed so that $k_{1}<k_{2}$.

In the following corollaries, we retain the notation introduced above, and assume throughout that $S$ is an umbilic-free surface $C^{4}$-immersed in a $C^{\infty}$ 3-manifold of constant sectional curvature. Proofs are omitted wherever an obvious computation yields the result.

COROLlary 1. If $H^{\prime}$ is constant on $S$, then wherever $\varepsilon_{1} \varepsilon_{2} \neq 0$, $2\left(\varepsilon_{1} \varepsilon_{2}\right)^{2} K\left(\Lambda^{\prime}\right)=2 \varepsilon \varepsilon_{1} \varepsilon_{2} K\left(\mathrm{I}^{\prime}\right)+H^{\prime}\left(\beta^{2}-4 \alpha \gamma\right)(\beta+2 \gamma H)\left|\operatorname{grad} k_{i}\right|^{2}, i=1,2$.

In particular, wherever $K \neq 0$,

$$
K\left(\mathrm{II}^{\prime}\right)=(H / K) K\left(\mathrm{I}^{\prime}\right)+\left(H^{\prime} / 2 K^{2}\right)\left|\operatorname{grad} k_{i}\right|^{2}, \quad i=1,2 .
$$

If the immersion of $S$ were smoother, the next result would follow directly from (3) and (5). As is, one must compute that the bracket in (6) vanishes if $\varepsilon \equiv 0$ on $S$.

Corollary 2. If $\varepsilon \equiv 0$ on $S$, then $\Lambda^{\prime}$ is flat wherever $\varepsilon_{1} \varepsilon_{2} \neq 0$.

The next corollary states little more than can be found in [7] and [13].

Corollary 3. If $H$ is constant on a surface $C^{2}$-immersed in a $C^{\infty} 3$-manifold of constant curvature, then away from umbilics, $\Lambda^{\prime}=-H \mathrm{I}^{\prime}+\mathrm{II}^{\prime}=H^{\prime} \mathrm{I}$ is flat. If the surface is minimal, $\mathrm{II}^{\prime}$ is flat and positive definite wherever $K \neq 0$.

Proof. Because $H$ is constant, coordinates can be chosen on the surface so that the immersion is $C^{\infty}$. (We thank Joel Spruck for this fact, and the reference [3].) Apply Corollary 2 with $\alpha=-H, \beta=1$ and $\gamma=0$. On a minimal surface, $H \equiv 0$ and $K \leqslant 0$, with umbilics characterized by $K \equiv 0$.

Corollary 4. If $K \equiv$ constant on $S$, then $\Lambda^{\prime}=-K \mathrm{I}^{\prime}+\mathrm{III}^{\prime}=2 H^{\prime} \mathrm{II}^{\prime}$ is flat.

Proof. Apply Corollary 2 with $\alpha=-K, \beta=0$ and $\gamma=1$. (Since $K \neq$ $0, \Lambda^{\prime}$ is never degenerate.)

REMARK 1. In [9] we show that $H^{\prime} \mathrm{I}^{\prime}$ is flat (away from umbilics) under the hypotheses of Corollary 3, and that $H^{\prime} \mathrm{II}^{\prime}$ is flat under the hypotheses of Corollary 4. 
COROLlary 5. If $\alpha \geqslant 0$ and $\gamma \geqslant 0$ are constants, then wherever $\varepsilon=\alpha \pm 2 \sqrt{\alpha \gamma} H+\gamma K \neq 0$ on $S$, the curvature of $\Lambda^{\prime}=\alpha \mathrm{I}^{\prime} \pm \sqrt{\alpha \gamma} \mathrm{II}^{\prime}+$ $\gamma \mathrm{III}^{\prime}$ is given by $K\left(\Lambda^{\prime}\right)=K\left(\mathrm{I}^{\prime}\right) / \varepsilon$.

Remark 2. If $K \equiv 0$ in Corollary $5, \Lambda^{\prime}=\alpha \mathrm{I}^{\prime}+2(\gamma H \pm \sqrt{\alpha \gamma}) \mathrm{II}^{\prime}$ and $\varepsilon=\alpha \pm 2 \sqrt{\alpha \gamma} H$. In case $H$ is also bounded, one can take $\alpha / \gamma$ so large that $\varepsilon$ never vanishes. Similarly, if $H \equiv 0$ in Corollary 5, $\Lambda^{\prime}=(\alpha-\gamma K) \mathrm{I}^{\prime} \pm 2 \sqrt{\alpha \gamma} \mathrm{II}^{\prime}$ and $\varepsilon=\alpha+\gamma K$. In case $K$ is also bounded, one can take $\alpha / \gamma$ so large that $\varepsilon$ never vanishes.

Corollary 6. Wherever $K \neq 0$ on $S, K\left(\mathrm{III}^{\prime}\right)=K\left(\mathrm{I}^{\prime}\right) / K$. In particular, wherever $K \neq 0, \mathrm{III}^{\prime}$ is flat if and only if $\mathrm{I}^{\prime}$ is flat.

Proof. Apply Corollary 4 with $\alpha=0$ and $\gamma=1$.

COROLlaRY 7. If $\alpha$ and $\gamma$ are nonnegative constants, $\Lambda^{\prime}=\alpha \mathrm{I}^{\prime} \pm 2 \sqrt{\alpha \gamma} \mathrm{II}^{\prime}+$ $\gamma \mathrm{III}^{\prime}$ and $\varepsilon=\alpha \pm 2 \sqrt{\alpha \gamma} H+\gamma K$ never vanishes on $S$, then

$$
\iint K\left(\Lambda^{\prime}\right) d A_{\Lambda^{\prime}}=(\operatorname{sign} \varepsilon) \iint K\left(\mathrm{I}^{\prime}\right) d A_{\mathrm{I}} .
$$

In particular, if $K$ never vanishes on $S$,

$$
\iint K\left(\mathrm{III}^{\prime}\right) d A_{\mathrm{III}^{\prime}}=(\operatorname{sign} K) \iint K\left(\mathrm{I}^{\prime}\right) d A_{\mathrm{I}^{\prime}}
$$

Proof. The element of area associated with a quadratic form is the square root of the absolute value of its determinant. Thus $d A_{\mathrm{I}^{\prime}}=d A_{\mathrm{l}}$, and one checks easily that $d A_{\Lambda}=|\varepsilon| d A_{\mathrm{I}}$. Corollary 5 yields

$$
\iint K\left(\Lambda^{\prime}\right) d A_{\Lambda^{\prime}}=\iint K\left(\mathrm{I}^{\prime}\right) \frac{|\varepsilon|}{\varepsilon} d A_{\mathrm{I}}=(\operatorname{sign} \varepsilon) \iint K\left(\mathrm{I}^{\prime}\right) d A_{\mathrm{I}} .
$$

REMARK 3. While the Gauss-Bonnet theorem is still valid [1] for indefinite metrics like $\Lambda^{\prime}$ in Corollary 7 , it is of only limited value. For if a compact surface possesses an indefinite metric which is nowhere degenerate, then it is topologically a torus or a Klein bottle, with zero Euler characteristic $\chi[2, \mathrm{p}$. 112]. On the other hand, we apply Corollary 2 only to umbilic-free surfaces, so that, once again, $\chi=0$ follows if the surface is compact.

Corollary 8. Wherever $K \neq 0$ on $S$,

$$
\begin{aligned}
K\left(\mathrm{II}^{\prime}\right)= & (H / K) K\left(\mathrm{I}^{\prime}\right) \\
& \pm(1 / 4 K)\left[\frac{d k_{2}}{d s_{1}}\left(\frac{d}{d s_{1}} \ln \left|\frac{k_{1}}{k_{2}}\right|\right)+\frac{d k_{1}}{d s_{2}}\left(\frac{d}{d s_{2}} \ln \left|\frac{k_{1}}{k_{2}}\right|\right)\right]
\end{aligned}
$$

where \pm is the sign of $k_{2}-k_{1}$.

COROLlaRY 9. If $k_{1}=c k_{2}$ for a constant $c \neq 0,1$, then $K\left(\mathrm{II}^{\prime}\right)=$ $(H / K) K\left(\mathrm{I}^{\prime}\right)$ wherever $K \neq 0$. 
REMARK 4. On an all-umbilic surface $C^{4}$-immersed in a $C^{\infty} 3$-manifold of constant sectional curvature, one can check directly that $K\left(\mathrm{II}^{\prime}\right)=$ $(H / K) K\left(\mathrm{I}^{\prime}\right)$ wherever $K \neq 0$. (See [11].)

COROLLARY 10. Wherever the greater principal curvature is a constant $c>0$ and $K \neq 0, K\left(\mathrm{II}^{\prime}\right) \geqslant H K\left(\mathrm{III}^{\prime}\right)$ on $S$. Wherever the lesser principal curvature is a constant $c>0$ and $K \neq 0, K\left(\mathrm{II}^{\prime}\right) \leqslant H K\left(\mathrm{III}^{\prime}\right)$ on $S$. If $c<0$, the inequalities are reversed.

Corollary 11. Suppose $S$ is an umbilic-free surface of revolution $C^{4}$-immersed in a complete, simply-connected $C^{\infty} 3$-mainfold $\Re$ of constant sectional curvature. Let $k_{1}$ denote principal curvature in the direction of meridians, $k_{2}$ in the direction of parallels, and let $\mp$ be the sign of $k_{1}-k_{2}$. Then

$$
K\left(\mathrm{II}^{\prime}\right)=(\mp H / K) K(\mathrm{I})+(1 / 4 K)\left[\left(d k_{2} / d k_{1}\right) \ln \left|\frac{k_{1}}{k_{2}}\right|\right] \text {. }
$$

Proof. There is no problem in identifying $S$ as a surface of revolution, since $\mathfrak{R}$ is a model for Euclidean or non-Euclidean geometry. Along any parallel, $k_{1}$ is constant. By the Lemma in $\$ 4$ below,

$$
K\left(\mathrm{I}^{\prime}\right)=\left[\operatorname{sign}\left(k_{2}-k_{1}\right)\right] K(\mathrm{I}) \text {. }
$$

Thus (7) reduces to (8) wherever $K \neq 0$.

3. On some special surfaces there is a natural way to distinguish two families of principal directions which are everywhere orthogonal (as on surfaces of revolution which avoid the axis of revolution). One then usually indexes the principal curvatures so as to assign one to each family of directions (although $k_{2}-k_{1}$ may then change sign on the surface). When this is done, it is natural to consider signed skew forms I", II" and III" defined (even at umbilics) as follows. Choose lines-of-curvature coordinates $x, y$ locally so that $k_{1}$ is principal curvature in the first distinguished direction $d y \equiv 0$, and $k_{2}$ is principal curvature in the second distinguished direction $d x \equiv 0$. If $\mathrm{I}=E d x^{2}+G d y^{2}$, let $\mathrm{I}^{\prime \prime}=-E d x^{2}+G d y^{2}, \mathrm{II}^{\prime \prime}=-k_{1} E d x^{2}$ $+k_{2} G d y^{2}$ and III" $=-k_{1}^{2} E d x^{2}+k_{2}^{2} G d y^{2}$. Wherever $2 H^{\prime \prime}=k_{2}-k_{1} \neq$ 0 , this yields $\mathrm{I}^{\prime \prime}= \pm \mathrm{I}^{\prime}, \mathrm{II}^{\prime \prime}= \pm \mathrm{II}^{\prime}$ and $\mathrm{III}^{\prime \prime}= \pm \mathrm{III}^{\prime}$ with \pm the sign of $H^{\prime \prime}$. Under the hypotheses of our Theorem, one can compute $K\left(\Lambda^{\prime \prime}\right)= \pm K\left(\Lambda^{\prime}\right)$ for $\Lambda^{\prime \prime}=\alpha \mathrm{I}^{\prime \prime}+\beta \mathrm{II}^{\prime \prime}+\gamma \mathrm{III}^{\prime \prime}$ wherever $\varepsilon \neq 0$ and $H^{\prime \prime} \neq 0$. Moreover, so long as $C^{4}$ coordinates $x, y$ of the sort described above exist in the neighborhood of an umbilic, it can be shown that $K\left(\Lambda^{\prime \prime}\right)=K\left(\mathrm{I}^{\prime \prime}\right) / \varepsilon$ at the umbilic, if $\varepsilon \neq 0$ there.

4. The formulas in $\$ 2$ become more useful if $K\left(\mathrm{I}^{\prime}\right)$ can be conveniently computed. The next result helps in very special cases. (See $\$ 3$ for the definition of $I^{\prime \prime}$.)

LEMMA. Let $S$ be a surface $C^{4}$-immersed in a Riemannian 3-manifold. Throughout the domain of $C^{3}$ geodesic lines-of-curvature coordinates $x, y$ such that 


$$
\mathrm{I}=d x^{2}+G d y^{2}, \quad \mathrm{II}=k_{1} d x^{2}+k_{2} G d y^{2},
$$

one has $K\left(\mathrm{I}^{\prime \prime}\right)=-K(\mathrm{I})$ with $\mathrm{I}^{\prime \prime}=-d x^{2}+G d y^{2}$.

Proof. Simple computations [13, p. 136] yield $K(\mathrm{I})=\left(G_{x}^{2}-2 G G_{x x}\right) / 4 G^{2}$, while $K\left(I^{\prime \prime}\right)=\left(-G_{x}^{2}+2 G G_{x x}\right) / 4 G^{2}$.

REMARK 5. On surfaces of revolution, the meridians are a family of geodesic lines-of-curvature. Thus $\mathrm{I}^{\prime \prime}$ is well defined at all points off the axis of revolution, with $K\left(\mathrm{I}^{\prime \prime}\right)=-K(\mathrm{I})$ if the profile curve is $C^{4}$. In $E^{3}$, there is a family of geodesic lines-of-curvature globally defined on cylinders (and locally defined on surfaces with $K \equiv 0)$.

REMARK 6. The formula $K\left(\mathrm{I}^{\prime}\right)= \pm K(\mathrm{I})$ does not always hold. The right helocoid [12, pp. 61-62, 89] in $E^{3}$ has $K(\mathrm{I})=K \neq 0$, but $\mathrm{I}^{\prime}=\left(1 / H^{\prime}\right) \mathrm{II}$, and $K\left(\mathrm{I}^{\prime}\right) \equiv H \equiv K(\mathrm{II}) \equiv 0$. Thus, while the Lemma shows that $K(\mathrm{I}) \equiv 0 \mathrm{im}$ plies $K\left(\mathrm{I}^{\prime}\right) \equiv 0$ for a surface in $E^{3}$, the converse is false.

REMARK 7. The Lemma shows that a correspondence between surfaces preserving $K(\mathrm{I})$ and $K\left(\mathrm{I}^{\prime}\right)$ need not be an isometry. Just take any map of the pseudosphere in $E^{3}$ upon itself which is not an isometry. Similarly, an isometry between surfaces preserving $K\left(\mathrm{I}^{\prime}\right)$ need not be a congruence. Just take an isometry between two pieces of the pseudosphere which is not the result of a Euclidean motion. (See [12, pp. 148-149].) Finally, an isometry between surfaces need not preserve $K\left(\mathrm{I}^{\prime}\right)$. Just take the standard isometry between the catenoid and the right helicoid. (See [12, pp. 120-121].)

\section{REFERENCES}

1. S. S. Chern, Pseudo-Riemannian geometry and the Gauss-Bonnet formula, An. Acad. Brasil Ci. 35 (1963), 17-26. MR 27 \#5196.

2. N. J. Hicks, Notes on differential geometry, Van Nostrand, Princeton, N.J., 1965. MR 31 \#3936.

3. E. Hopf, Über den functionalen, insbesondere den analytischen Charakter der Lösungen elliptischer Differentialgleichungen zweiter Ordnung, Math. Z. 34 (1931), 194-233.

4. T. Klotz, Another conformal structure on immersed surfaces of negative curvature, Pacific J. Math. 13 (1963), 1281-1288. MR 28 \#32.

5. \#646. , Surfaces harmonically immersed in $E^{3}$, Pacific J. Math. 21 (1967), 79-87. MR 38

6. A complete $R_{\Lambda}$-harmonically immersed surface in $E^{3}$ on which $H \neq 0$, Proc. Amer. Math. Soc. 19 (1968), 1296-1298. MR 38 \#1641.

7. T. Klotz and R. Osserman, Complete surfaces in $E^{3}$ with constant mean curvature, Comment. Math. Helv. 41 (1966/67), 313-318. MR 35 \#2213.

8. T. K. Milnor, Complete, open surfaces in $E^{3}$, Proc. Sympos. Pure Math., vol. 27, Part 1 , Amer. Math. Soc., Providence, R.I., 1975, pp. 183-187. MR 52 \#1533.

9. The curvature of $\alpha \mathrm{I}+\beta \mathrm{II}+\gamma \mathrm{III}$ on a surface in a 3-manifold of constant curvature, Michigan Math. J. 22 (1975), 247-255.

10. $\_$Restrictions on the curvatures of $\Phi$-bounded surfaces, J. Differential Geometry 11 (1976), 31-46.

11. M. Spivak, Some left-over problems from classical differential geometry, Proc. Sympos. Pure Math., vol. 27, Part 1, Amer. Math. Soc., Providence, R.I., 1975, pp. 245-252. MR 51 \#6863. 
12. D. J. Struik, Lectures on classical differential geometry, Addison-Wesley, Reading, Mass., 1950. MR 12, 127.

13. J. A. Wolf, Surfaces of constant mean curvature, Proc. Amer. Math. Soc. 17 (1966), 1103-1111. MR 34 \# 765.

School of Mathematics, Institute for Advanced Study, Princeton, New Jersey 08540

Department of Mathematics, Rutgers University, New Brunswick, New Jersey 08901 